\title{
WHOSE BODY IS IT ANYWAY? UNDERSTANDING BODY IMAGE IN PREGNANT AND POSTPARTUM WOMEN
}

\author{
Devika Kapoor
}

\author{
Tata Institute of Social Sciences, Mumbai, India
}

\begin{abstract}
Pregnancy is a phase where a woman's body goes through a plethora of inevitable physical changes which continue to linger in the postpartum period, the study aimed to explore how young pregnant women and new mothers are appropriating or resisting dominant discourses on the body and obesity as well as how they constitute their experiences within available discourses. A qualitative inquiry in the form of in-depth interviews were undertaken to understand the various negotiations women make and a feminist lens was used to understand the narratives of these women. The study was conducted on 7 pregnant and 7 postpartum women residing in Mumbai. It was found that women felt less pressurized to maintain the thin ideal during pregnancy; however they did experience the pressure to live up to the pregnant woman ideal. The postpartum women faced tremendous pressure to lose postpartum weight and conform to the thin ideal. Subsequently, they reported high levels of body dissatisfaction since they now had to live with a new bigger body. Apart from media, family members and peers, the role of spouse in shaping body image was considered of utmost importance by pregnant as well as postpartum women. The study also tried to understand the nature of body modification techniques and grooming routines during pregnancy and postpartum period. The inquiry revealed that body modification strategies saw a dip during pregnancy, however women who employed some fitness activity pre-pregnancy continued to employ pregnancy safe activities like pregnancy yoga. During postpartum, women did employ weight loss strategies, but most of them were unable to continue doing so either because of exhaustion or lack of time.
\end{abstract}

Keywords: Body Shaming, Feminist, Body Ideals, Body Dissatisfaction

\section{Introduction}

Body image relates to self perception about one's own body . (Cash, 2002). The formation of body image starts extremely early in childhood. Body image relates to self-perception about one's own body .This self-picture is influenced by a person's beliefs, attitudes, experience and ideals followed by society. Accordingly, one may have a positive or negative body image. Body image is a complex construct and is closely linked with the identity of individuals and believed to influence their behaviors. A negative body image may lead to certain behavioral changes like withdrawal from social engagements or loss of self-esteem while a positive body image may give a person confidence and motivation to succeed. How a person begins to have a negative or positive body image is a matter of interest. The size and shape of the body have come to increasingly operate as a market of personal, internal order or disorder - a symbol for the emotional, moral or spiritual state of the individual.

The first attempt to understand the idea of body image can be traced back to over nearly a century. Body image has been studies through various perspectives. The role of body and body image has been theorized by many perspectives, from psychologists to philosophers to physicians have tried to understand the concept of body image. (Thomas F. Cash, 2002). Recently, a new culture is evident, that of "body culture" and various disciplines, including sociology has now come up with their theories and discoveries on the body and how it is relates to the individual. The body is revived in order to analyze and understand issues like self-image and embodiment. Women are considered the ones who have the highest rates of body dissatisfaction and body image problems. "Idealized images of beautiful women are a major factor affecting young women's personal ideas and body image; other influential factors include peers and family and the perceived preference of the opposite sex" (Casanova 2004: 289) Feminists who explored the effects and nature of female beauty ideals reported that the beauty ideals were a way of objectifying women. The beauty ideals have undergone massive transitions with every passing decade. The ideals during the previous century were that of a more fuller woman, with large 
breasts, and a curvaceous figure. However the industrial revolution bought about major shifts in the beauty ideals all over the world. Attractiveness and beauty had become synonymous with being thin. A wave of thinness was experienced all over the world. Feminist have argued that this unrealistic image of what a female body should look like has managed to create anxiety and negative body image in women.

Evolution of body as a concept: Body image is an equivocal concept, and the array of issues that come under this umbrella term have also altered over time. In the initial years on 1990, body image was seen as the concern of neuropathy. Therefore, the studies during that time focused on how an individual perceives her/her body and a distorted body image was seen as an aftermath of brain damage" (Grogan, 2007)

Multi-dimensional understanding of Body Image: Schilder's writing also throw a light on the complex nature of body image, given the fluidity and multifaceted nature as tapped through research. There continue to be some underpinnings that have tackled the test of time. It is a well established fact that body image is an integral part of human experience. (Cash, 2002). Though there is a growing body of research on body image, there are still areas within the discipline that remain untapped. Majority of the literature in body image tends to adopt a rather pathological lens that focuses on disordered / deregulated eating. It thus becomes necessary to look at body image as a collection of experiences that is shaped by various environmental and internal factors. Since it has been well established that body image is a socially constructed phenomenon that affects individuals at a personal level, it is vital to the various sources that influence the development of body image. The Indian female ideal has undergone massive transition post globalization. One of the biggest change that the Indian society has seen post globalization is the change in beauty ideals. Globalization has managed to redefine the ideals of beauty for both women and men. The exposure to highly photo shopped images of models have successfully infiltrated the beauty ideals of Indian society. The Indian woman today doesn't compare herself to the traditional Indian standards, she compares herself to the western Ideal. A fair skinned girl who is tall, slim, has long hair which are mostly colored or highlighted, narrow waist and proportionate hips (Zimik, 2016). It is vital to recognize that the construction of one's body image is a complex interplay of myriad factors. Previous researches have shown that various factors in a person's environment influence the way someone thinks about their own body. The most commonly studied influences are:

Familial influence: Developmentally speaking, family is the first unit of social interaction in a child's life. Perceived parental pressure has been portrayed as one of the foremost sociocultural variables identified with the development of negative body image. (Rheanna N. Ata, 2006). Parental feedback, and specifically coordinate remarks in accordance to appearance, have been linked disordered eating and body dissatisfaction in both males and females (Baker, 2000). "Parental teasing has also been shown to be associated with body dissatisfaction and disordered eating, as has encouragement to diet" (Keery, 2005). The parent's body satisfaction or dissatisfaction reflects in the child, and their qualities and standpoints in regards to physical appearance and attractiveness are consolidated readily by their children. (Rodgers, 2009).

Peer Influence: A vast body of literature in the area of factors affecting body image has concluded that peers are the most prominent source of influence during adolescence and continue to be so even in early adulthood. Recent studies examining the impact peers have on the formation of body Image concluded that, during adolescence, peer group impacts young boys and girls much more than media. (Hargreaves, 2006)

Influences of romantic partners : As an individual gradually progresses through adolescence, the influence of peers start to become less profound. During young adulthood and emerging adulthood, it is the romantic partners that have the most resonating impact on the development of body image. Intimate romantic relationships are multidimensional and involve a number of perceptions regarding self and the romantic partner. (Swami, 2009) Research also shows positive links between relationship wellbeing and emotional heath and in turn body image such as the association between steadfast romantic relationships and greater overall joy and fulfilment in life (Goins, 2010)

Media Influence: "The influence of media on the development of body image has been studied in lengths by many researchers. It is a well-established fact that media continues to impact body image across life span but with fluctuating intensity. Where, its influence is most pronounced in adolescence but it dips slightly as in individual matures chronologically. The impact of media has managed to sweep across gender. Both male and female seem to be equally impacted if not equally affected by media. Different sources of media like newspapers, T.V, magazines, movies have a huge consumer base and a much wider impact. It is only obvious that with such daily and continuous encounters with various forms of media, its influence on formation of body image and identity is a given." 
Both men and women have internalised these beauty ideals, although the effects are more profound in case of women, that is, they express greater body dissatisfaction as a result of internalising the thin ideal. (Jones, 2004)

\section{Feminist View of Body}

The 1990s witnessed a fundamental disparity stemming from the feminist scholars on the concepts related to beauty and appeal. Western feminists have expressed trepidation about the role of women's bodies in limiting their freedom since the first wave of the feminist movement. In 1914, in the midst of the rights listed to assure women's equality in the United States also included "the right to ignore fashion". The second wave of the feminist movement during the 1960s and 1970s, the role of the body was heavily inspected. Second wave feminist authors critiqued and linked patriarchy and hegemony to the kind of dressing styles women were expected to follow, which was more ornamental while the men's dressing was rather convenient they also critiqued the expectations form women to "appear a particular way." Feminist activists questioned societal expectations that a woman should perpetually try to 'improve' and 'modify' their bodies and appearance through regular routine like body hair removal, wearing makeup, and dieting in order to lose weight. While thinness might symbolize control and success in the minds of women who try to emulate the ideal, its unrealistic nature means that women' s energies are wasted" (Bordo, 2004). Women as a group are devalued in a patriarchal, heterosexist society and a focus on appearance helps endure women's low status. Regardless of minor role alterations for women, there is still a disempowering focus on the body.

\section{Objectification Theory}

One feminist theory related to the body that has received a lot of empirical attention is 'objectification theory', proposed by Barbara Fredrickson and Tomi-Ann Roberts. Objectification theory is heavily influenced by feminist philosophy arguing that the ubiquitous sexual objectification of women leads women to internalize the objectification, reflected in self-objectification. Self-objectification puts women at risk for negative emotional experiences including body shame, which, in turn, increases the risk for eating disorders, depression, and sexual dysfunction. Much research has now linked self-objectification with body shame, which mediates scores on measures of eating disorders, depression, and sexual dysfunction. In addition to the thinness expected of women, it has been found that there is increased sexualisation of women in the culture. In one recent study, it was found that about one-half of the advertisements in a variety of magazines featured women as sexual objects, and the sexualisation of women in magazines has increased since the 1980s.

\section{Body Image in adulthood}

Most researches in the domain of Body Image has been in with people belonging to 18-25 years of age. (Grogan, 2007) Work that has looked at body image concerns in older adults has produced some interesting findings. The idealised slim and slender body type is usually paired with youth. Women particularly are expected to try to maintain a rather youthful appearance, since youthfulness for women is considered more attractive and is also valued across many parts of the world. Many researchers have noted a 'double standard' of ageing, where women are judged more severely than men in terms of physical attractiveness as they show signs of ageing. Gender role expectations network with age and gender to affect attractiveness judgements (Sorell, 1981). Signs of ageing in men are seen as something that makes them look 'distinguished', while women are often judged in terms of physical attractiveness rather than in terms of abilities or experience signs of ageing may be seen negatively both by others and by themselves. Research has shown that ratings of physical attractiveness decreased with age for both men and women, but that the decline was steeper for female than for male targets.

\section{Body Image in Pregnancy}

A woman goes through huge changes during pregnancy. She experiences physical, mental and emotional changes. During the course of her pregnancy, a woman will gain significant amount of weight that are coupled with other noticeable physical changes increased breast size, larger waist size, widening of the pelvis and hips, and both temporary and permanent skin changes. Even a woman's hair, nails, and shoe size are often altered. At no other time is a woman's weight and diet such a focus to herself, family, physicians, and even complete strangers. Thus it is not surprising that pregnancy is often marked by significant changes in body image satisfaction - both for the better and for the worse (Thomas F. Cash, 2002). With the significant amount of 
changes to a woman's body that push her farther from the ideal body shape than ever, body image researchers have accounted positive impacts associated with pregnancy. Women typically forego dietary restriction and rigid body image ideals in favour of a more nurturing role (Clark, 1999). It was also found that pregnant women have less dietary restraint, rate themselves as less overweight, have lower drive for thinness, and when BMI (body mass index) is controlled for, much lower body dissatisfaction scores than non-pregnant women. However, pregnant women's ideal body size generally remains the same as non pregnant women's ideals. This suggests that women may be able to make specific cognitive and/or behavioural adjustments to their body image while pregnant, despite the presence of underlying negative body image and eating schemas. The transition to a more positive body image maybe a hard task and may take time. Several studies have suggested that women's perceptual and subjective body image tends to be more positive at a later stage in pregnancy than the initial months, when women first start gaining weight and thus experience a change in their body shape. It may be that women are more uncomfortable and extremely new with their shape when they do not clearly appear pregnant or when the experience of gaining weight is novel. Later in pregnancy, women do receive positive reinforcements and reactions for their changing appearance, mainly- increased weight. This motives them to take up and even celebrate the maternal role rather than perceiving themselves as overweight. (Cash, 2002).

\section{Body Image in Postpartum}

Pregnancy and the postpartum year are often viewed as periods of tumultuous change for mothers. Although almost all aspects of a woman's life are altered by the birth of a baby, concerns about weight gain and getting back to pre-pregnancy shape are among the most frequently expressed by postpartum women.

Strang and Sullivan have shown that body image dissatisfaction peaks in the postpartum period. Most women continue to look pregnant in the first few weeks following birth, and in the first few months the majority of new mothers continue to weigh more than their pre-pregnancy weight. During pregnancy this weight gain may have been perceived as a positive sign of the baby's health and development. However, it is generally not experienced as acceptable after giving birth, and quick weight loss is the expected goal. Some degree of postpartum body image dissatisfaction may be due to unrealistic weight loss expectations. The majority of new mothers expect to return to their pre-pregnancy weight and shape by 6 weeks postpartum. In reality, most weight loss occurs during the first postpartum year, with decelerating loss occurring as the year progresses.

\section{Objectives of the Present Study}

- $\quad$ 1. Idea of beauty : Understanding internalized concept or notions of beauty or attractiveness

- 2. Evaluating/Understanding the way women view their appearance during Pregnancy and Postpartum

- $\quad 2.1$ Retrospective understanding of their body/ appearance prior to pregnancy and post pregnancy

- $\quad 2.2$ Understanding attitudes towards physical changes during pregnancy and postpartum

- 3 . Understanding perceived influences in the way women look at their appearance during and post pregnancy

- 4. Exploring the various body modification or fitness methods women employ during and post pregnancy

\section{Methods}

With an attempt to completely understand the effect that pregnancy and motherhood have on how women view their bodies, a qualitative research design was chosen. The qualitative approach was contributory in helping capture the respondents' embodied experiences in relation to their bodies, this method which disallowed the minimizing of body image to a static. This method was especially helpful in understanding the culture specific beliefs and practices linked with pregnancy and motherhood.

\section{Tool}

The qualitative data was collected using an interview guide. The interview guide had approximately 30 questions and a few questions were added depending on the responses of the participants. The interview guide had three broad sections namely. Pre pregnancy, Pregnancy and Post pregnancy. The questions were designed primarily in English. These questions were framed in such a manner that they focus on the Domains of inquiry. Such as understanding their concept of beauty, their ideals and it's evolution, their body image development 
trajectory. Factors that they think influenced their development of body image- both positive and negative. The effect that puberty had on their body image, what were the various body modification strategies that they used throughout their life, the reasons for the same. The questions later focused on were they reconstructing their body image during pregnancy. The various cultural practices and beliefs around pregnancy that influenced their body image. How did their dressing style change during pregnancy and how did they adjust to that change. What were the various grooming routines they followed otherwise and did that change during pregnancy. What effect did pregnancy have on their relation with their partners. The third part of the interview guide focused on what are the various physical changes they went through as a result of having delivering a child. What was it like for them to make meaning of it. What did they think that their body would look like as opposed to what did it actually look like once they delivered. Do they plan to lose weight, if yes then when would they do so and what will be the strategies. What are the various cultural beliefs regarding motherhood and mothering in their families. How did parenthood affect their relationship with their partners.

\section{Analysis}

All the interviews were transcribed and a manual line by line coding was done, later, common themes were identified and a thematic analysis was done.

\section{Sample}

The total strength of the quantitative sample was 10 of which there were 5 pregnant women and 5 postpartum women who are currently residing in Mumbai.

The inclusion criteria for pregnant participants were that the participant should be under the age of 35 and over the age of 25 . She should be voluntarily continuing pregnancy to term for the first time. She should at least be in her second trimester of her first pregnancy. She should belong to a higher socio economic status.

Women who are expecting multiple births were also included in the sample.

The inclusion criteria for a postpartum participant were: The participant should be under the age of 35 and over the age of 25 . She should be a first time mother. Her child should not be more than 12 months. She should belong to a higher socio economic status. The pregnancy should be a voluntary pregnancy.

\section{Sample characteristics}

The current study is a qualitative inquiry of the data gathered from 7 pregnant and 7 postpartum women. A detailed account of their characteristics is as follows-

\section{Pregnant Participants.}

All the pregnant participants were approached personally either through private clinics and/or pregnancy yoga classes all around Mumbai, India.

The age range of the pregnant participants was 25-32 years and the mean age was 27 . The range for moth of pregnancy was 4-11 months and the mean was 8 months.

Out of the pregnant participants, 5 of them were full time employed, none of them were part time employed and 1 of them was self-employed and 1 of the participant was a home-maker.

The mean personal income of the pregnant group was 43,000/ month and the mean family income of the group was $1,10,000 /$ month.

\section{Education Qualification}

Out of the pregnant participants, 4 of them had a post graduation degree, 3 of them had a completed their graduation and none of them had a diploma. 


\section{Type of marriage}

5 out of 7 people had a love marriage and the remaining 2 had an arranged marriage. The range for "years of marriage" was 2-7 years and the mean was 3 years.

\section{Postpartum participants}

Majority of the postpartum participants were contacted personally either through a paediatric clinic or day care centres all around Mumbai.

The age range of the post partum participants was 25-32 years and the mean age was 29 . The range for "postpartum month" was 4-11 months and the mean was 7 months.

\section{Employment status}

Out of the 7 postpartum participants, 5 of them had a full time job of which 2 participants were currently employed full time and 3 of them were on their maternity leave. 1 of them was part time employed, one of them were self-employed and none of them was a home-maker. The mean personal income of the postpartum group was $47,000 /$ month and the mean family income of the group was $1,00,000 /$ month.

\section{Education Qualification}

Out of the 7 postpartum participants, all of them had a post graduation degree.

\section{Type of marriage}

3 out of 7 people had a love marriage and the remaining 4 had an arranged marriage. The range for "years of marriage" was 4-8 years and the mean was 5 years.

\section{Findings and Discussion}

\section{Understanding internalization of body Ideals}

Body ideals have undergone tremendous transformations since the last century. Looking at the historical antecedents of what was considered beautiful a while ago, we realize that the female beauty ideal has seen huge shifts. When women possessing features like large breasts, thick arms and wide hips were considered attractive. However, this ideal underwent a lot change post industrial revolution. The ideal female body or ideal look of a woman is when a woman is thin, tall, has long hair, has large breasts, has a clear skin and symmetrical features. Attractiveness and beauty become synonymous with being thin post the industrial revolution in several western counties. A wave of thinness was later experienced all over the world. However, the reason for the shift towards a thin ideal has been unexplored. There has been no research to understand the reason why the shift happened in the first place. However, it is important to note that the shift in Indian beauty standards was much later. The shift in Indian beauty standards only happened post globalization where India saw a one way exchange of beauty ideas.

Pregnancy is known to be a time when there is a sanctioned departure from the typical female ideal. Although, this does not render women pressure free. There seems to exist an ideal for the pregnant body too. The pregnant body ideal is typical of a "big enough" baby bump, a slightly fuller body that the feminine ideal. Large breasts and lastly, a face that has "pregnancy glow". There also exists an ideal for a postpartum woman or a new mother. An ideal look of a new mother is constructed to be that which is more or less similar to the feminine ideal. In other words, the new mother is expected to be thinner than her pregnant self, have an even skin tone and is expected to possess a body that is similar to her body pre pregnancy.

Feminist theories suggests that women are often subjected to unrealistic body ideals right from childhood, this often results in internalization of these ideals. (Murnen, 2012). Internalization can be understood as a process through which the characteristics, beliefs, feelings and attitudes that are present and propagated by the culture an individual is part of becomes assimilated into the individual's identity and sense of self. When a person is unable to attain the internalized ideal, it results in tremendous body dissatisfaction. Body dissatisfaction, 
defined as negatively evaluating and feelings of shame over one's body weight, shape, or muscularity (Cash, 1994), is currently a widespread experience among women across the world ( McCabe, Ricciardelli, Waqa, Goundar, \& Fotu, 2009; Mellor et al., 2009; Swami, Frederick et al., in press). The reason why we tend to follow the ideal is because we experience a lot of positive reinforcement for following the ideal. The social expectancy theory thus say that we tend to follow the ideal because we are encouraged to do so and thus we are also positively reinforced by the society for doing so. Since in the current study, the two ideals that came up very strongly were: "The Thin Ideal" and "Skin Ideal". Further discussion will focus only on these ideals.

\section{The Thin Ideal}

As mentioned earlier that the feminine ideal has undergone a massive transformation. The "thin ideal" has been now internalized by many women. Internalization of the thin ideal refers to the extent to which an individual believes this ideal as a viable and necessary way that she, herself, should look (Thompson, 1999). Its is important to discuss the difference between internalization and awareness; one can be aware of the thin ideal without internalizing, or cognitively adopting, the thin ideal. Unfortunately, the ideal is impossible to attain. Thus, women who try to maintain the thin ideal themselves our generally dissatisfied and are left feeling disappointed with themselves and with their bodies when they do not achieve A great deal of research has found that internalizing the thin ideal can predict both body dissatisfaction and eating pathology in young women. One of the most potent sources linked with the dissemination of the thin ideal is media. (Park, 2005) noted that the body size of women portrayed in mass media has been steadily getting smaller.

Another medium through which the process of internalization takes place is through approvals by the significant people in one's life, such as: Family members and Peers (Kandel, 1987). The known channels in this process are comments or actions that serve to support and perpetuate this ideal like, criticism or teasing regarding weight, encouragement to diet, and glorification of ultra-slender models These sources communicate expectations concerning the benefits of thinness, such as increased social acceptance, and these expectations likely play a key role in the propagation of this ideal. Such internalization may result in negative body image. Past research reveals internalization of the thin-ideal and subscription to gender-based discourses, indicated by the uptake of body surveillance and self-silencing, are significant variables effecting negative body image.

In the current study, when women were asked about what they considered as an ideal look for a woman, they answered that an ideal woman would be the one who is Well groomed, has good hair, is tall has symmetrical features, has clear skin and lastly, has a fair skin. These findings clearly depict how the participants have internalized the feminine ideal. In the following example, a participant narrates the feedback she received since she did not follow the feminine ideal.

B.W says "I have always been on the heavier side, right from my childhood. Since I am so tall, I used to look huge, almost like a boy! Every time I went to take a dress, the shopkeeper would look at me and refuse, saying that the dress isn't available in my size. I used to feel very bad when this used to happen. Then I decided to lose weight. I began skipping meals, walking $7 \mathrm{Kms}$ a day and just eating cucumber. I lost $12 \mathrm{Kgs}$ of weight in 3 months. Everyone was happy with my weight loss. Everyone was happy, except me. Because, I continuously wanted to lose more weight. I think I was happier when I was fat."

The above example clearly shows the struggle saturated journey of a "non-conforming" individual to try and match the ideal. It is also important to notice how other people responded to the weight loss. Their response is direct reflection of the larger dominant discourse that surrounds the narrative of a "desirable body". The responses received from the significant others in the participant's life, validate the previous discussion that relates to the reasons why we follow the ideal.

\section{The thin Ideal during pregnancy}

Pregnancy is a time when a woman's body does alter dramatically, this alteration is largely a deviance from the larger thin ideal present in the society, with lingering bodily changes continuing into the postpartum period. Studies have indicated that women tend to adapt to the weight changes during pregnancy positively. It is an established fact that pregnant women do report that there they feel less pressure to maintain a certain weight while they are pregnant, Simultaneously, other scholars have argued that women are very aware of their bodily changes during pregnancy and that such changes invoke conflicting feelings regarding weight. More specifically, many researchers have discovered that a good fraction of women feel "uncomfortable" about their appearance and size during pregnancy. 
M.G said, “Although I am happy that I am pregnant, I don't want to gain too much weight. I read lot regarding pregnancy and weigh gain, because I did not want to gain weight unnecessarily"

Previously researchers have found that depending on women's weight before pregnancy, they felt more or less comfortable with weight gain — those being heavier feeling more at ease with their changing bodies during pregnancy. In some cases "pregnancy was therefore used as a legitimate reason for gaining weight. However, this still had to be within acceptable boundaries" (RAIL, 2012)

In the words of C.P, "You know, although people stop commenting on your weight gain, they are not very forgiving when you gain weight on other places except your stomach and maybe have larger breasts"

The above example depicts that although the thin ideal is not "encouraged" during pregnancy, it is not completely relaxed. Thus to make a generalized comment saying that "the thin ideal" doesn't exist during pregnancy would be fallacious.

\section{Thin Ideal during Postpartum}

The thin ideal tends to revive in the postpartum period, where new mothers face unrealistic demands to boomerang to their pre-pregnancy body almost instantaneously.

One of the leading causes of postpartum depression is body image dissatisfaction among new mothers. (O'hara, 2009). One of the reasons why this happens is because the media constantly depicts and celebrates the weight loss of new mothers. The Indian media and the media abroad has coined the term "yummy mummy" which is an extremely objectifying term that means a new mother who is "back to being attractive and fit". Recently, there has also been a rise in gym programs that focus on "post natal weight loss". It is not surprising to learn that body image concerns spike the most during pregnancy (Strang, 1985)

R.K said, "No, I am definitely not satisfied with the way I look right now. I have to lose weight in order to look good. People tend to ask me why I haven't lost weight even after nine months. I don't know what to say..."

The above narration highlights the pressure put on postpartum women to lose weight. It is also, important to note, how the participant reported that she did not know how to respond to the question. She perhaps is feeling guilty for not losing weight. This exhibits the kind of anxiety women are made to experience when they do not conform to the thin ideal.

\section{Skin Ideal}

Majority of the participants, reported skin as being one of the aspect that makes a person look attractive. The major change in the beauty ideal was deception of fair skin as a symbol of desirability. The Indian audience was exposed to a number of advertisements that propagated unattainable beauty standards such as being tall, thin and most importantly, being fair skinned, akin to the European "White" ideals. A closer look at the findings of the current study revealed that majority of the women from considered having fair skin as one of the aspect of attractiveness. Furthermore, participants have reported how being of a darker shade was not considered a good thing.

P.K says "Well, the ideal woman in our culture is never dark. My sister is darker than me, so she used to always get remarks that were negative. People used to say that, "Saawali hai, toh kam se kam zara tayaar hokar raha kar" (Since you are dusky, at least groom yourself)."

This example shows the huge amount of value attached to fair skin. Since there is somewhere an understanding among people that skin tone is not something that can be altered, they suggest compensatory strategies for making up for the lack of it.

\section{Skin Ideal in Pregnancy}

Pregnancy comes with its own ideal for skin. As mentioned earlier, during pregnancy the one thing that is emphasized the most the "pregnancy glow" there are certain skin ideals that tend to come up during pregnancy. 
H.K said, "Yes, I have started to get stretch marks now, but I don't like it. So I have now started to use oil to prevent further stretch marks. See, it doesn't look good when you wear sleeveless and if you have stretch marks on your hand"

In the above example it can be clearly seen how the participant does not feel comfortable about the change in her skin as a result of pregnancy since a "clear skin" is more desirable.

P.K says, "My sister sent me this oil from Australia, she used it during her pregnancy and did not get any stretch marks, and I used it too and that's why I did not get any stretch marks anywhere.

This example shows how family members help in maintaining the skin ideal during pregnancy.

\section{Skin Ideal during Postpartum}

The skin ideal during the postpartum is similar to the skin ideal propagated in the pre-pregnancy period. However, there are some aspects of the skin that are typical of the postpartum period. Some of the ideals are: toned non-saggy skin, even skin tone without any pigmentation and void of any surgical scarring.

E.G said, "The only reason I continue to wear a saree is because I had a normal delivery and did not have any marks from c-section or stretch marks too. If I had them I would have not worn saree till they vanish. It doesn't look good"

The above example is a clear depiction of internalized postpartum skin ideal. The participant mentions that the only reason she would choose to wear a saree is because she has no surgical scaring or stretch marks. She goes on to say that if she would have them she would never wear a saree because scars and stretch marks are not considered aesthetically pleasing. This shows that how tabooed natural phenomenon like having stretch marks or scars are. This also is a reflection about the lack of dialog regarding what actual motherhood looks like and what an actual body of a new mother looks like. Internalization of the "Pregnant Body" ideal. As mentioned earlier that there exist a ideal for the pregnant body ideal that is different from the thin ideal. However, having said that, it is not to be confused that pregnant women suddenly start feeling positive about their bodies. Though they do not compare themselves against the "general idea", they do seem to have a "pregnant ideal"

When the participants of the current study were asked what an ideal body looks like, the majority reported. That a pregnant woman is the one who has a Big bump but a small body, is well groomed, has glowing skin, has an overall rounded body. The findings of the present study draw a similar trend.

A.P said "An ideal pregnant woman is someone who has a nice and round belly. She should carry herself with grace. You know like how, Genelia D’Souza(Indian Actress) was! So cute looking. Even though she was pregnant, only her stomach was visible, her hands and legs were exactly the same as before pregnancy. I remember she had done this interview where she was wearing a nice hair band she has worn a nice dress. She was looking so cute!"

In this example the participant interestingly mentions the nexus of both the ideals. She mentions that the said celebrity was looked cute because she had a "visibly big bump" which is borrowed from the pregnant ideal and the fact that her hands and legs were exactly the same as before which is borrowed from the thin ideal. This examples captures the struggle of negotiation women who are pregnant go through where they are now expected to conform to the pregnant ideal holding on to the thin ideal. A.P's narrative also helps us understand how media has managed to create an ideal image for pregnant women.

Another aspect that was briefly mentioned in the internalised skin portion is the internalisation of the pregnancy glow.

In the present study, most of the participants in the agreed to this being as one of the features of an ideal woman. However, they reported that they never felt the glow themselves, it was always other people who said that they were glowing.

E.G said "Honestly, I never felt that I was glowing. In fact I thought I looked even more tired than usual. But other people such as my relatives and colleagues used to say that I am glowing. Although, I never felt it, eve on 
my baby shower when I was so tired and irritated, people said that I was glowing. I'm sure that was not the case"

It is thus important to note that "pregnancy glow" is so deeply interlaced with the appearance of a pregnant woman and pregnancy, that even though women do not experience themselves, they consider it to be an ideal. Medically, the only possible explanation as to why some women experience "pregnancy glow" is because of the extra secretion of oil. ((Ed.), 1994) Thus it is important to understand that the various contestable notions surrounding pregnancy and physiological changes may create dissatisfaction in pregnant women and thus put them at a risk of developing a negative body image during pregnancy. (Anton, 2000)

It is also important to understand that although women say that they feel that the society approves of the weight gain, they did mention a different kind of scrutiny they underwent that was typical of this period. Very recently, there has been a new emphasis on the transition to parenthood in the popular media, with increasing attention to celebrities' "bumps". They experienced scrutiny with regard to the size of their baby bump. Thus they faced a phenomenon called "Bump shaming". This means that women are questioned on the size of their bump. They are either shamed for having a "larger than normal" bump or "smaller than normal bump". Women in the present study unanimously mentioned that they experienced at one such incident where they were questioned regarding the size of their baby bump thus facing bump shaming for not attaining the ideal.

\section{Internalization of the new mother ideal}

The new mother ideal, as discussed before is similar to the general women ideal. When the participants of the present study were asked the features of an ideal new mother, the participants reported aspects like, large breast, less body fat, lesser stretch marks and body more or less like pregnancy. It is interesting to see how the aspects such as lesser stretch marks, body more or less like pre pregnancy. Women from the both group of the current study considered aspects like less stretch marks and body becoming more or less like pre pregnancy a part of the new mother ideal. It is also medically impossible to go back to the exact pre pregnancy bodies. However a possible explanation for why women think so is that there is no reference point available to the larger audience expect media. Media has always depicted fabricated images of new mothers. Not only has the media disseminated unrealistic standards for new mothers, it has actively shamed women who have broken the norm.

Like Like M.K says "Aishwarya Ria took a lot of time to get back to her pre pregnancy figure, The media did left no chance to shame her for doing this." She further added how this helped her "be okay" with her body, she said "I think she proved to be such an inspiration to me and all my other friends. She did something no other celebrity did." Though this is an example of how a celebrity acted as a protective factor, there were other participants who expressed their discontent with celebrities losing weight immediately post delivery.

J.A said, " During pregnancy, I thought that I will lose at least ten kgs immediately after the delivery. But that did not happen! I was slightly disappointed. Because, whenever a celebrity comes after the delivery, she is so thin. It's like they have a magic wand!" It is clear that women tend to think that once they deliver, their body will be more or less like pregnancy and when that doesn't happen, women face a lot of emotional turmoil and great amount of body dissatisfaction. (Strang, 1985). Thus it can be established that all these ideals are internalized by women and the failure to meet the ideal creates higher body image dissatisfaction.

\section{Understanding the way women look at themselves during pregnancy and postpartum}

\section{Pregnancy}

Women have always been expected to maintain a certain feminine ideal; that of the slim, attractive, youthful body. Conversely, pregnancy has been considered a time when this surveillance from the people reduces. This is partially related to historical constructions and view of the pregnant body as needing increased rest and less vigorous activity, for fear of harming the mother and child in turn. Furthermore, the pregnant woman has been "expected" to focus inwardly on the child, rather than outwardly on her appearance. Thus, as women transition to the pregnant body, there has been lowered expectation to uphold the slender feminine ideal. (RAIL, 2012). Lately, however, there has been an increased expectation to uphold an ideal feminine body even during pregnancy, by holding a certain (relatively slim) shape and size, and by 'bouncing back' more quickly after giving birth. 
Representations of the pregnant body in popular culture are now common than ever as celebrities are featured in magazines during pregnancy and again shortly after giving birth. Some reason that these representations may increase pressure on pregnant women, serving a panoptic function, as the women in the magazines are toned and lean, showing "weight" only in the stomach area during pregnancy and leaving no sign of pregnancy postpartum.

Moreover, there is increased expectation that pregnant women uphold a 'yummy mummy' body by managing to be attractively dressed, well groomed, and fit throughout pregnancy and the postpartum period. Dworkin and Wachs as well as Jette have argued that exercise is being positioned as a means to keep the body in shape during pregnancy and 'regain' the pre-pregnancy body more quickly afterwards (O'hara, 2009). Simultaneously, increasing medical literature is highlighting 'risks' allied with obesity during pregnancy for the mother and child. (Cockey, 2005). Many scholars have identified a dominant obesity discourse that parallels slenderness with health, and within which weight control is emphasized as an individual matter of choice and will power. Other scholars have suggested that such discourse leads to the stigmatization of those who do not uphold the bodily norm. (RAIL, 2012). Feminist scholars have discussed in detail the strong impact these rigid bodily expectations have on women as they can lead to disordered relationships with the body and a lower quality of life. (Tiggemann, 2001) Accordingly, some researchers have raised concerns about possible and similar negative effects of an obesity discourse that qualifies the slim body as the 'normal' body. Others have discussed the possible impact of a media emphasis that constructs the slim body as the "healthy and beautiful" body. Furthermore, feminist researchers have stressed the pressure this bodily ideal may put on women during pregnancy, leading to further anxiety about weight gain during pregnancy and dangerous weight control strategies. (Anton, 2000).

Through this objective, the present study purports to gain a deeper understanding of whether young pregnant women are appropriating or resisting dominant discourses on the body and obesity as well as how they constitute their subjectivities within available discourses.

Exploring pregnant women's images and representations of their body, some scholars have found that they are reoriented away from the feminine slender norm. Researchers have proposed that body changes and weight gain are understood as an indication of foetal growth and signs of a new role as mother (Chang, 2006). It has also been found that women emphasize the functionality of their body (as producing a new life) over the aesthetic requirements of feminine embodiment. These authors have suggested that the changing idea of the body from ornamental to functional may lead some women to feel comfortable with their body during pregnancy. Like P.K said, "I simply stopped worrying about my weight gain during pregnancy. I gained a lot of weight but I did not see it as problematic, I used to think that I am doing all this for my child. I think all women should stop dieting during pregnancy. It's just matter of 9months, you have your whole life to diet."

When the participants of the current study were asked as to what were the features that they had during pregnancy. Majority of the women reported that, they had overall rounded body, large breasts, chubbier face and swelling of feet. Participants mentioned that they had an overall rounded body and even chubbier face. Interestingly, none of the features that the participants reported of having matched what they considered as ideal for pregnant women.

Gaining weight during pregnancy during pregnancy is perceived as acceptable; however, only to a certain point and in certain places. Earle(1990), has discussed the various concerns that pregnant women have regarding weight, including when they will begin to look pregnant, where changes on their bodies will happen, and the ease with which they will return to pre-pregnancy weight. The transitory nature of pregnancy also influences the comfort that some women feel with gaining weight. Bailey (2001), for instance, has commented that some of the pregnant women understand pregnancy as a temporary state and were therefore anxious about their changing bodies and fearful of trying to lose weight post-partum. Finally, a number of scholars have observed a some levels of distress towards the body and weight gain during pregnancy. In brief, there seems to be two very different sets of findings so far on women's experiences and representations of their body during pregnancy: some pointing to women being comfortable with their pregnant body and others suggesting anxiety and worry in relation to this body.

While the narrative of the women display the contradictions and paradox inherent in the women's constructions of their pregnant body, the feminist discourse analysis aims to highlight how women navigate dominant or resistant discourses surrounding the pregnant body. 
Some scholars have claimed that pregnancy defies modernist understandings of the body and is characterized as a "series of biological transitions over which the mother-to-be has little control". Indeed, participants do simultaneously recite this alternative discourse of the uncontrollable pregnant body. On a number of instances they mention that their body "is going to do what it wants" and that listening to their body is vital during pregnancy, including when to rest and eat. Some also mention that their body is going to gain how much it wants, where it wants, regardless of what they do. Thus, at times, the participants of the current study do appropriate the dominant obesity discourse, but at other times resist constructions of a controllable body during pregnancy. As mentioned earlier, expectations to hold the feminine ideal may be relaxed during pregnancy.

However, the women's narratives point to conflicted feelings about this 'break' from conventional femininity. On the one hand, the women resist the overall view that they need to uphold a certain appearance and many mention that this is a time when they do not care as much about how they look; they discuss dressing for themselves instead of others and wearing clothes in which they feel comfortable. The women in the current study recite resistant discourses whereby they feel free to enjoy their bodies and forget about the ideal feminine form. On the other hand, many of the participants also mention feeling nervous about losing the body with which they are comfortable. Moreover, although some of the women display awareness of the social construction of their 'normal' body, this does not prevent them from feeling anxious about losing that body. The women worry about how much weight they are going to gain and where they will gain it. The participants discuss the increased physical presence of their body and the external gaze that renders them more aware of their body. They also recall injunctions heard from outsiders that reproduce discourses of beauty and femininity during pregnancy (e.g., how much and where pregnant women should gain weight) as well as derogatory comments towards pregnant women who gain "too much" or who are "fat." The women are aware of the public scrutiny over their pregnant body and they resist the idea that this gives outsiders excuses to judge them.

B.W says "When I had not announced my pregnancy to other people at my office, they always looked at me oddly because I was gaining weight".

A.R says “It's nice that I'm pregnant, but I don't look like myself anymore, my clothes don't fit me. I know this is temporary, but still..."

\section{Postpartum}

The postpartum period is considered to be the time in a woman's life when a female's body undergoes a lot of changes. Body image dissatisfaction seems to be the highest during the post-partum period when women have to now live with a "new" body. New mothers are again expected to conform to the feminine ideal, of being thin and slender. Popular media acts as another agent for maternal scrutiny of women's bodies. Media images of women provide graphic representations of the ideal form of motherhood; mothers then compare their own bodies to these images and often view themselves critically and undesirably (Garner, 1980). The shift to motherhood involves substantial changes in a woman's life, perhaps none so public and rapid as the changes to her body as she grows with pregnancy. While most people are aware of the physical changes of pregnancy, many who have not undergone the transition themselves are less aware of the short-term and enduring changes in a woman's body. Given the culture of thinness-as-beauty that pervades our society today, it seems that any changes to body weight or shape might impact one's sense of self esteem and well being.

Most of the studies have found out that women in the postpartum period may be additionally vulnerable to body image concerns. One of the major reasons for this vulnerability is that women while they are pregnant are encouraged to gain weight and "behave pregnant" by restricting body movements and involving in less rigorous activities. However, post the birth of the child, postpartum women are scrutinized for their pregnancy weight gain and there is an added pressure of child care involved. It is only natural that upon childbirth, women will not immediately go back to their pre-pregnancy body. Once they realize that, they are likely to harbor negative feelings towards their body. (Tiggemann, 2001). When women in the postpartum group were asked as to how did their bodies actually look like one month after delivery, they reported that they had a larger waist size, large breast size, dry skin and had excessive hair fall. It is astonishing to witness that none of the characteristics matched the characteristic of the "ideal" new mother. One can only imagine the kind of disillusionment and a burst of distress that postpartum women undergo.

In a general way, social factors, such as social support networks, may change as a new mother assumes new roles and identities. In addition, one's culture dictates the beauty ideals to which one is compared, even for postpartum women. Even if individuals do not themselves internalize the ideal or if they understand the ideal to be unrealistic, they still feel that others will judge them by these standards (e.g. Milkie, 1999) 
In the most overwhelming words of M.K “ My $8^{\text {th }}$ month postpartum body is an apology to everyone who expected a well-toned body from me after my delivery"

E.G said "I never thought that I will still wear my maternity clothes 6 months after delivery. Nothing fits me anymore and I remain too tired to work-out. I tried doing yoga, but I cannot get up early morning and go to yoga classes because I am up the whole night taking care of him (her 6 month old son) I feel like I am getting fatter every day. I cannot even diet because I am breast feeding. It's like I have no place to go"

It is almost like when a woman is pregnant, she has a sense of reversibility. While they thought that they are going to get back to their pre-pregnancy body almost instantaneously. Discourses of femininity and bodily control are increasingly emphasized to control the body post-pregnancy. Almost all the postpartum participants expressed their worry about regaining their pre pregnancy body after giving birth. More specifically, many of the participants are concerned about body changes that are counter to the feminine ideal such as stretch marks, and excess fat. After childbirth, most women do not immediately return to their pre-pregnancy size or weight, and many women never regain their pre pregnancy shape. Additionally, research focusing on postpartum weight loss shows that, many women experience feelings of grief and disappointment in regards to their body after pregnancy (Jenkin \& Tiggemann 1997; Johnson \& Williamson, 2004).

After their pregnancy, Collett (2005) says women might constantly remind people or inform them they just had a baby, so as not to confuse her changed body with one of laziness or "letting go." As if explaining or justifying their body weight. This is explained by the social expectancy theory, where a well toned or a lean body is looked as if the person is in control and can "handle" things.

Similar to women's account of pregnancy, postpartum women also reported dramatic bodily changes and a sense of having a 'new' body after having a baby. Post-birth, women described thinking about their body similar to early pregnancy; during pregnancy once the pregnancy 'showed' women would reason 'I am pregnant, not fat" however, in early pregnancy and again in the postpartum women were not made to experience the social sanction to be large and gain weight, since they were not "visibly" pregnant. In the postpartum despite women being aware that they had recently given birth, most women spontaneously noted that the fact that this was not apparent to the wider community contributed to worsened body attitudes. For example, one mother stated: " $I$ had a legitimate excuse for my weight while I was pregnant. Now that I am no longer pregnant, I no longer have that excuse"

In pregnancy, women seemed to attach more or less positive connotations to the various body changes such as: large tummy, swollen feet, weight gain because they attributed it to the growth of the baby thus women were able to see the positive function of those changes. However,

at postpartum, women did not talk of their body in this purposeful way, and most participants seemed shocked and surprised that they did not return to their pre-pregnancy shape quickly. M.K said "As I look at myself in the mirror, I do not recognize myself. My stomach was sagging down, I then started wearing a belt so that my stomach comes back to its normal size. I had never imagined myself in this shape"

There was another set of response sometimes given by the same participant when asked about their feeling related to physical changes. Although, the women were dissatisfied with their new bodies and thus felt more attractive. They also reported that they feel that it is not the time to think about their bodies now. They reported that they think that they should take care of their child/Children and not think about themselves because their baby "needs them" It was almost women felt guilty about thinking about themselves now that they are mothers.

J.A said "I don't think I should focus on myself right now. This time is for my baby and I should make sure that all her needs satisfied rather than focusing on how my body is looking no matter how much I want to lose weight." This statement reflects how women make these negotiations that are often a result of how they have been socialised to motherhood. Ideals of contemporary motherhood now prescribe new set of tasks beyond the first shift of work and the second shift of household labor and child care. There is now a required third shift of bodywork (Wachs, 2004) Articles define the postpartum body as chaotic, disorderly, and stressful New mothers are said to be "primed for anxiety", and bodily "discoveries" are framed as sinful, out of control, shameful, or evidence of a lax body that must be quickly corrected. Women are described as being "caught off guard" while asserting that "I hadn't expected to feel out of control, nor had I known that pregnancy and childbirth would be a completely body-changing experience" (spring 1998 issue, 101). 
Thus it can be concluded that though women feel largely dissatisfied with their bodies, in the postpartum period, either because of the unpreparedness of what their bodies are going to look like or either because of the unrealistic demands that are placed on women to appear a certain way since women no longer experience the legitimacy of gaining weight unlike in pregnancy. Subsequently, one of the reasons why women feel unhappy with their bodies postpartum is because how they are socialized to motherhood, where they have internalized to concept of giving up their bodies because "having a child demands so" (Strang, 1985) Finally, the most important reason of body dissatisfaction among young women is the false images of what a new mother should look like that media portrays.

\section{Understanding the perceived influences in the way women look at their appearance in pregnancy and postpartum}

Women reported a number of factors that shaped their body image some of the common factors that women talked about are Peers, Family members, Spouse, Media, Pregnancy blogs, Pregnancy book, their doctors and even mobile application to certain extent. This section discusses how various aspects influences the construction body image across three phases of the participants

In the pre pregnancy period women reported that while growing up, it was the comments of family members that mattered to them the most. They recollected the incidents while growing up where family members passed comments on their appearance C.P said "I remember that they used to compare me to my sister. My sister is darker than I am, so every time people used point that out. I remember feeling bad when these comparisons happened. But then I had this picture in my head that I am fairer than my sister." This statements proves that how comments received from the family members lead to the development of some aspects of body image. Participants also recalled the first time that they noticed how they looked and the factors that led them to this understanding.

M.K said " The first time I noticed how I look was when I was in college. This was because my friends passed some comment on how I look and I did not like the person they compared me to. So I remember coming home and looking in the mirror and checking if I look like that person. Since I did not like that person, I started to dress differently, so that I look different." This example clearly shows how peers help play In important role in formation of body image and what are the various changes a person tries to make to their appearance on receiving feedback from others.

Home and family environment have been found to be an extremely potent influence when it comes to young people's body image; both explicit weight-related comments and implicit parental modeling may have adverse effects on adolescents (Neumark-Sztainer, 2010). Negative dialog concerning body image from family members, including critical comments, teasing, and encouragement to diet, have been known to be linked with the development of body image disorder and eating disorder symptomatology (Curtis, 2014). B.W said "I remember this one particular incident clearly, when I was standing on the railway station and my aunt passed a comment on my weight and skin tone. Although she had never told me anything regarding my body before, so hearing that from her came as a shock to me. She never told anything about my body, but that one thing really affected me" Kichler and Crowther (2009) found that even infrequent or rare comments made by family members can have a negative impact. Bailey and Ricciardelli (2010) came to the conclusion that young women who receive negative weight-related comments tend to compare themselves to others negatively and are more likely to develop Body image dissatisfaction. Neumark-Sztainer et al. (2010) also found that family teasing about weight is common, with more than half the young women in their study reporting being teased by family members in the previous year, and in no instances was parent weight-talk or family.

The role of the family and family members is that of an extremely crucial one, even during the pregnant and postpartum phase. Prior research has shown that if pregnant women get a positive and a more accepting environment at home, they are less likely to be affected adversely by the physical changes during pregnancy.

Apart from the already mentioned influences, the one influence that stood out in the construction of body image especially during pregnancy and postpartum period was Spousal influence. All the participants unanimously mentioned that their spouse had a huge impact on their body image. In addition to the direct influence of bodyfocused teasing and comments by family and peers, research has shown that the negative feedback received from spouse also may affect the emergence of body dissatisfaction. It is important to consider the role of social relationships in determining women's body satisfaction. Because women are socialized to "accept weight as a defining aspect of their value" (Charlotte N. Markey, 2004) 
In this study we found that some women reported about experiences where their partners had made negative comments about their bodies during pregnancy and some participants reported a perceived shift in the way they considered their own attractiveness in the relationship. B.W said "Spousal support and what they think of your body matters A LOT! I will tell you this one incident where in my 7th month, my husband was looking at me and then I asked him, "What are you looking at?" he said "You are looking so different, you have gained so much weight" I told him "You are not supposed to make me feel this way and say all this about my body. After he said that I started looking at my old photos and felt that I have gained too much weight. I started crying. Later, when he came and apologized to me, and said that he likes me the way I am, I felt better"

A.R said " He is very supportive and is like a friend to me, and we have a very nice relationship, but even then, while I was gaining weight, I thought I was looking unattractive. I also feared that he has an affair with someone since we we have decided to not have sex till the time I am pregnant and for a few time after that too. one day, I asked him that does he think that I have become unattractive because of the weight gain? He said that no, I am still attractive and he still loves me that really helped me be happy throughout my pregnancy"

It is thus important that husbands might actually act as a protective factor their wives from the glorifiedextremely-thin ideal by explicitly communicating to their wives their body preferences. Because some research indicates that feeling accepted, valued, and supported by meaningful others may render women more resilient to social messages about body image. Spouses can play an important role in efforts aimed at increasing women's body satisfaction (Markey, Markey, \& Birch, 2001).

\section{Spousal influence in postpartum}

Spousal influence on body image increases in the postpartum period. Research has shown that how women think that their partners perceive them has a huge impact on the body image of women. Spouses play an extremely important role, in the postpartum period. It has been noted that postpartum is a time when the intimacy between the couple goes down because of various reasons, one of the most important reason being that women do not feel comfortable about their postpartum bodies and thus they avoid being intimate with their partners. How the husband perceives the changes in his partners' body is extremely vital. If spouses take the changes in their partners' positively and communicate it to the partner, it leads to better acceptance by the wife, of the physical changes that she has gone through and thus helps in the development of a positive body image.

J.A said "I used to feel very unconfident regarding my body after the baby. One day my husband noticed that I wasn't wearing any good clothes so, he asked me as to why am I not wearing my good clothes, so I said that I don't look good in them anymore. What he said next, really made me very happy. He said that "I like you no matter what size you are and you look good in everything you wear",

The above example thus shows how the positive comments of the spouse acted as a protective factor towards creating a negative body image.

\section{Body Modification and beautification techniques employed during pre pregnancy, during pregnancy and post pregnancy}

As discussed earlier, there is a tremendous pressure to be thin and follow the thin ideal among women As a result, women tend to undertake certain physical activity to alter their body weight and shape. The participants of the current study were asked if they undertook any physical activity prior to pregnancy in order to regulated weight and shape, and the findings revealed that most of the participants wanted to undertake a physical activity but were unable to undertake but the ones who did undertake some weight regulation strategies were going to the gym, yoga, and dieting. The fact that most people reported that they wanted to undertake a physical activity but they did not only shows the extent to which people feel the need to alter their existing bodies, perhaps because of the dissatisfaction that they feel with their body and the compulsion to follow the thin ideal.

M.B said, "I always felt that I should lose weight not much but 5-6kgs but I never had the time to join any classes"

The findings of the present study indicate that the only time when majority of the women felt tremendous need to lose weight was once during adolescence and then before their wedding. Research has shown that most people dissatisfied with their bodies and try to modify it in adolescence (Anton, 2000) The known reason is that 
an individual's body does undergo a lot of changes during puberty and these changes may not be perceived in a positive light most of the time.

M.B said "Yes, I did feel like dieting and losing weight when I started going to college my body had become bulkier than before and I was getting teased from my friends, so I thought to lose weight and become thin. At that time, my parents wouldn't give me money to join the gym or any classes because they did not approve of the weight loss, so the only option I had was that of dieting. So I went on a diet and lost weight back then"

From the above example it is clear how there was a struggle between the family members and the participant and their opposing ideas regarding weight loss, where she wanted to lose weight but the family members did not support the idea. It also highlights how the participant then chose to exercise her autonomy on her body, which is by dieting. (Tiggemann, 2001) in his study has noted that dieting is the most widely used strategy employed by adolescence girls and is one of the reasons for development of eating disorders.

The pre-wedding weight loss.

All the participants of the current study mentioned that they at least attempted the pre- wedding weight loss. This is a recent fad that has developed in the past few years. It is not surprising to learn that there are various pre-weight loss programs that are now offered .

B.W said, "Well yes, I did got for the pre wedding weight loss, where they basically make you lose five kilos in twenty days. I wanted to look good on my wedding day and that is the reason why I went for that. See, you get married only once so you need to look the best on you wedding day. Also, people take so many photographs of you during your wedding so it is better to look good in those photographs because you have to look best at that time."

In B.W's narrative, one can clearly see the way she has internalized the thin ideal. There are instances where she relates being thin with being beautiful. The narrative also brings out the pressure that women these day face in order to "look like an attractive bride", this brings out the fact that there is also pressure to look a certain way at you wedding.

A.R said, "Yes yes, I did go for the weight loss program. Actually, I had also done a pre-wedding photo shoot so I had actually lost a lot of weight for that because I wanted to look good in those pictures. I had planned for a very long time that I will lose weight before the shoot. I had become so thin you know..." after saying this sentence, she removed her phone and showed a couple of photos to share with me her "weight loss success story". After viewing the photos herself once again, she seem to recount her "thin days" after a few seconds of silence, she said “ But, I don't look like this anymore” and took a deep breath almost, feeling a longing to go back to her pre-pregnancy body. A.R was pregnant and was now compelled to follow the pregnant ideal and although the thin ideal was slightly relaxed, it was evident that she had some ambivalence regarding the pregnancy weight gain.

\section{Body modification techniques during pregnancy}

Research in the area of body modification during pregnancy has shown that, women tend to not adopt any weight loss strategies during pregnancy, even though they may not always be okay with the weight gain. They refrain from indulging into any such activities since they believe in that doing so will harm the child (Cockey, 2005). When participants of the current study were asked regarding the fitness activities they undertook during pregnancy, findings revealed that they undertook activities like pregnancy yoga. The reason why they chose this activity was because they considered yoga safer than any other physical activity. Women believed that they should not undertake rigorous exercise since it could harm the child. Also, such exercises do not have a cultural sanction during pregnancy.

J.A says, "The moment I came to know I was pregnant, I stopped exercising because I did not want to risk my pregnancy"

B.W says “ I don't think that I will even think of exercising or going to the gym now that I am pregnant. I don't want to cause any harm to the child because of some stupidity of my own. I have my whole life to go to the gym. I will do so once I deliver" 
The above examples show how pregnant women in this study were not very open to the idea for taking any fitness activity during pregnancy. However, there seems to be some deviation to this too. It was noticed that in the current study, pregnant women who exercised prior to pregnancy did take up some sort of physical activity even during pregnancy. Although, the intensity of the activity and sometimes even the frequency decreased.

R. K said "See, I have been into fitness activities so, even during pregnancy I took up yoga although I did not go regularly because I used to feel tired. But I made sure that I at least do some sort of physical activity"

\section{Body Modification techniques during postpartum}

The postpartum period is said to be a period of elevated body concerns and body image dissatisfaction (Murnen, 2012). Also, this is the period where women wish to undertake fitness activities in order to reduce weight. Findings of the current sample revealed that women report that they are undertaking(postpartum women) and plan to undertake (pregnant women) activities such as yoga, walking or jogging and reducing fat content.

E.G said "I have started going for small walks. I cannot do rigorous gym exercised since I get tired, plus I cannot wake up early in the morning to do yoga because she (pointing towards her child) doesn't let me sleep at night. So, the only possible way I can lose some weight is through walking"

The above example shows how one of the reason why women choose a particular activity. It also shows how fatigue is a very real concern as to why women may sometimes not take up any physical activity.

R.K said, "I really want to lose weight, however I just cannot now. I cannot got to the gym because I have had a c-section, I cannot go for walks because there is a lot of joint pain, I cannot even diet because I am lactating and everything I eat is high in fat content because It increases milk supply"

Another interesting trend was observed. It was noticed that women in the current study who were undertaking some physical activity before pregnancy expressed more concern regarding the inability to lose the gestational weight.

\section{Beautification and beauty practices in the pre-pregnancy period}

Notions of femininity and beauty and their interdependence with the fashion and cosmetics industries have been very deeply ingrained with the feminine ideal. More and more beauty products have filled the market recently. Cosmetics and other beauty practices have been on a rise since the post globalization in India. The findings of a survey regarding product sale post globalization found out that the product which had recorded the highest sale was fairness creams, in India (Cardoza, 2009) Other beauty products such as hair removal, cosmetic surgery and laser treatments have infiltrated the feminine ideal.

There has been a feminist critique regarding the idea of beauty products and beauty practices. Radical feminists such as Liz Frost have argued against beautification practices and beauty products , She says that women's bodies are "inferiorised stigmatized.Within an overarching patriarchal ideology. For example, biologically and physiologically, women's bodies are seen as both disgusting in their natural state and inferior to men's body. (Frost, 1999). Cultural expectations about women in particular removing their body hair changed drastically in the early- to mid-20th century as clothing styles changed to reveal more skin (the legs and underarms in particular) and advertisements began to promote not hair removal products in general but competing products, effectively engraining the supposed regularity of body hair removal in the mind of the average consumer. (Trisolini, n.d.)

Thus, It can be seen that beauty standards have become more and more difficult to attain and the feminine ideal is now narrower than ever. When the participants of the current study were asked regarding their beautification techniques they employed in the pre pregnancy period, it was found that the beautification practices that we the most common were waxing and shaping their eyebrows. Other practices such as laser treatments, going for facials were not reported by the participants.

M.B said "I started waxing at the age of 15, this was my school farewell and that is the reason I went for waxing. All my other friends also started waxing at the same time. I In fact, it was my mother who took me to the parlor to get waxed. It was a painful experience, it still is. I personally do not like waxing but other force me to get waxed" 
The above example shows beauty practices start increasing in adolescence and in fact it rather becomes a norm which females are not only encouraged to follow but are appreciated for doing so. This examples also shows how significant others in the participant's life, disapprove her dislike for the practice and further force her to practice it.

\section{Beautification and beauty practices in the pregnancy period}

Beautification techniques in pregnancy do undergo various changes. Where women in the current study have mentioned that in there was a decline in the beautification practices because of the numerous cultural and to some extent scientific reasons. The findings of the current samples revealed that not many women employ beautification techniques such as waxing, shaping eyebrows, massages and anti-aging creams. Only a very small portion of women reported that they employed beautification techniques. This was because of a host of cultural reasons why pregnant women refrain from employing any beautification techniques during pregnancy.

E.G said " See, in my culture, pregnant women are not allowed to wash their hair for whole nine months except on their baby shower. So I could not even wash my hair, let alone beautification techniques. We do not wash our hair because as a tribute to the goddess of fertility. So, I was okay with not washing my hair."

The above example shows how religion and culture are one medium through which there are permissions or prohibitions of performing certain beautification techniques during pregnancy.

\section{Beautification techniques applied during post partum}

Beautification techniques do not change much in the postpartum period as compared to the pregnancy period. The results of the present study reveal that beautification techniques continue to dip in the postpartum period with the only exception being that massages and use of anti-aging creams increase during the postpartum period. Massage post pregnancy is an extremely culturally sanctioned beautification technique that is wide spread and even encouraged by family members and other significant people in their life. Although, there is a scientific as to why massages are recommended for post partum women, which is that of Massage is well known for relaxation, stress reduction, pain relief and other health benefits. Unique postpartum benefits include hormone regulation, reduced swelling, better sleep and improved breastfeeding. More advanced therapy helps restore your body to its pre-pregnancy condition, speeds healing and assists with C-section recovery. But women do not seem to know the significance of it, but rather view it as something that relaxes them.

P.K said, " No, I don’t know the scientific reason for it, I just relax during my massage, I find it very relaxing to my body."

\section{Conclusion}

The present study was conducted with an aim of understanding how women construct their body image in two distinct phases of their life, viz-a-viz pregnancy and postpartum. The study focused on understanding the internalized notion of beauty and attractiveness. When the participants of the current study were asked as to what are the physical features that make a women look attractive. Findings revealed that the physical qualities mentioned by the participants were extremely similar to the one that is propagated by media. The top few physical qualities were: Being well groomed, having good hair, being tall, possessing symmetrical features, having a clear skin and finally, having a fair skin. As mentioned earlier these aspects are very similar to the feminine ideal that is propagated by the media. Interestingly, the participants talked about a slim body and the thin ideal in much more in the postpartum period. The study further explored the internalized pregnant and postpartum ideals.

Women in the pregnancy experienced a respite from the social pressure to remain thin and maintain the thin ideal. They expressed that they felt the absence of scrutiny from the society to lose weight and maintain a thin body during pregnancy. However, this in no circumstance means that women did not face pressure of scrutiny from the society regarding their bodies. Although the women were not compelled to lose weight or have an overall thin body, they were expected to follow the pregnant ideal. The pregnant ideal as reported by the participants is the one of having a big baby bump but a thin body, having a clear and a glowing skin and being well groomed. Another unfortunate scrutiny that women felt during pregnancy was "bump shaming". Bump shaming is a phenomenon where the women face objectification because of the size of the baby bump. It is 
important to notice, that although women did not experience the social pressure to be thin or lose weight during pregnancy, they were expected to follow other aspects of feminine ideal.

The postpartum period was noted as the period where body image concerns were experienced the most. Women experienced tremendous societal pressure to go back to their pre-pregnancy bodies. A further inquiry into the new mother ideal revealed that, the new mother ideal is more or less similar to the feminine ideal. Women in the present study did report that they did feel extremely dissatisfied with their bodies because of the various physical changes their bodies underwent during pregnancy.

The next objective of the study was to explore the perceived factors that influenced the construction of body image across three phases- pre pregnancy, pregnancy and post pregnancy. During the pre-pregnancy phase, the factors that influenced were- Media, family members, peers. The factors that influenced the construction of body image during pregnancy were spouse, family members and doctors to a certain extent. During the postpartum period, women reported that they felt that their spouse played an extremely important role in the construction of body image. They also reported that apart from family members, media, they considered spousal support the most important during this period and believed that their spouses perception had the most impact during postpartum.

The study further aimed to study various body modification techniques across three phases, pre-pregnancy, during pregnancy and post pregnancy. It was found that in the pre pregnancy period, women did undertake a couple of physical activities such as going to the gym, restricted eating and going for yoga. The various body modification techniques that they used during this period were waxing shaping eyebrows, facials. However, during pregnancy women did not employ any rigorous physical activities, however some women, especially the ones that were undertaking some fitness activities pre-pregnancy reported employing some fitness activity even during pregnancy, like pregnancy yoga. The beautification techniques saw a dip in the pregnancy period because of various cultural and to some extent scientific reasons. During the postpartum period, women obviously experienced a lot of body dissatisfaction with respect to the increased body weight, however they reported that it became extremely difficult for them to employ many fitness activities either because of exhaustion or either because they weren't permitted to undertake few activities like restricted eating and going to the gym immediately because they were either lactating or were recovering from the c-section surgery. Thus, the study found out that although women did feel that the societal pressure to lose weight was slightly relaxed, they did face the pressure to live up to the pregnant ideal. In the postpartum period, they women again were expected to live up to the new mother ideal which was extremely similar to the ideal that they were supposed to follow prepregnancy.

\section{References}

(Ed.), R. V., 1994. Mayo Clinic Complete Book of Pregnancy \& Baby’s First Year. s.1.:New York, NY: William Morrow and Company, Inc.

Anon., 2015. Encyclopedia of Mental Health - Second edition. s.1.:s.n.

Anton, S. D. P. M. G. \&. R. J. R., 2000. Discrepancy between actual and ideal body images: Impact on eating and exercise behaviors. Eating Behaviors,. s.l.:s.n.

Bailey, 2001.

Baker, C. W. W. M. A. \&. B. K. D., 2000. Studying intergenerational transmission of eating attitudes and behaviors: methodological and conceptual questions.. Health psychology.

Baker, J. D. W. D. A. \&. S. C. (., 1995. Body image disturbance, memory bias, and body dysphoria: Effects of negative mood induction. Behavior Therapy,. s.1.:s.n.

Bandura, 1997. Social learning theory. s.1.:s.n.

Bartky, S. L., 1991. Femininity and domination: Studies in the phenomenology of oppression.. s.1.:Psychology Press.

Beller, A., 1977. Fat and Thin.

Bordo, S., 2004. Unberable Weight: Feminism, Western culture, and the body.. s.1.:Univ of California Press.

Bracey, R., 2011. Women in Kushan History. s.1.:s.n.

Caracelli, V. J. \&. G. J. C., 1997. Crafting mixed-method evaluation designs. New directions for evaluation. s.l.:s.n. 
Cardoza, R. P. 8. K., 2009. Melanin on the Margins: Advertising and the Cultural Politics of FairLightNhite Beauty in India.

Carruth, B. R. \&. G. D. L., 1990. Nutritional Issues of Adolescents Athletics and the Body Image Mania.. The Journal of Early Adolescence, pp. 10(2), 122-140..

Cash, T. F. \&. P. T., 2002. Future challenges for body image theory, research, and clinical practice. Body image: A handbook of theory, research, and clinical practice,509-516.. s.1.:s.n.

Chang, S. R. C. Y. M. Y. \&. K. N. J., 2006. I am a woman and I'm pregnant: body image of women in Taiwan during the third trimester of pregnancy..

Charlotte N. Markey, P. M. M. a. L. L. B., 2004. Understanding Women's Body Satisfaction:.

Chernin, K., 1983. Womansize: The tyranny of slenderness.. s.l.:s.n.

Clark, K., 1980. Feminine beauty. Weidenfeld \& Nicolson.. s.l.:s.n.

Clark, M. \&. O. J., 1999. The impact of pregnancy on eating behaviour and aspects of weight concern.. International journal of obesity, 23(1), , pp. 18-24..

Cockey, C. D. (., 2005. Obesity During Pregnancy. Nursing for Women's Health,.

Cohen, 1980. Fundamentals of Quantitative Research. s.1.:s.n.

Coomber, K. \&. K. R. M., 2008. The role of sisters in body image dissatisfaction and disordered eating. Sex Roles, 59(1-2).

Curtis, C. \&. L. C., 2014. Friends, family, and their influence on body image dissatisfaction. Women's Studies Journal, pp. 28(2), 39.

Darwin, C. (., 1888. The descent of man, and selection in relation to sex (Vol. 1). Murray.. s.1.:s.n.

Engeln, R. S. M. R. \&. W. H. (., 2013. Body talk among college men: Content, correlates, and effects.. In: Body Image, 10(3), 300-308.. s.1.:s.n.

Ford, C. S. \&. B. F. A., 1951. Patterns of sexual behavior.. s.1.:s.n.

Frost, L., 1999. Doing looks": Women, appearance and mental health. Women's bodies. Discipline and transgression,.

Garner, D. M. G. P. E. S. D. \&. T. M., 1980. Cultural expectations of thinness in women. Psychological reports,. Goins, L. B. (., 2010. Understanding men's body image in the context of their romantic relationships. s.l.:s.n.

Grogan, S., 2007. Body Image: Understanding Body Dissatisfaction in Men, Women and Children. s.1... Routledge..

Grogan, S., 2007. Body image: Understanding body dissatisfaction in men, women and children.. Routledge.: s.n.

Hanson, W. E. C. J. W. C. V. L. P. P. K. S. \&. C. J. D., 2005. Mixed methods research designs in counseling psychology. Journal of counseling psychology, 52(2), 224.. s.1.:s.n.

Hargreaves, D. A. \&. T. M., 2006. Body Image is for Girls’ A Qualitative Study of Boys' Body Image.. ournal of Health Psychology, 11(4), 567-576..

Jones, D. C. V. T. H. \&. L. Y., 2004. Body image and the appearance culture among adolescent girls and boys an examination of friend conversations, peer criticism, appearance magazines, and the internalization of appearance ideals.. Journal of adolescent research, 19(3), 323-339..

Kandel, D. B. \&. A. K. (., 1987. Processes of adolescent socialization by parents and peers.. International Journal of the Addictions.

Keery, H. B. K. v. d. B. P. \&. T. J. K., 2005. The impact of appearance-related teasing by family members.. Journal of Adolescent Health, 37(2),, pp. 120-127.

Kerr, C. a., 1986. Food for feminist thought.. The Sociological Review, pp. 34(3), 537-572..

Leifer, M., 1980. Pregnancy. Chicago Journals.

Leifer, M., 1984. Pregnancy. s.1.:s.n.

Mack, N. W. C. M. K. M. G. G. \&. N. E., 2005. Qualitative research methods: a data collectors field guide.. s.l.:s.n. 
Meltzer, A. L. \&. M. J. K., 2010. Body image and marital satisfaction: evidence for the mediating role of sexual frequency and sexual satisfaction.. Journal of Family Psychology, 24(2), $156 .$.

Mishkind, M. R. J. S. L. \&. S., 1986. The Embodiment of Masculinity: Cultural, Psychological, and Behavioral . In: American Behavioral Scientist. s.l.:s.n.

Moffitt, L. B. \&. S. D. M. (., 2011. Experiencing sexually objectifying environments: A qualitative study.. s.l.:s.n.

Murnen, S., 2012. feminist perspective on body image and appearance.

Neumark-Sztainer, D. B. K. W. F. S. H. P. J. S. M. \&. B. J. M., 2010. Family weight talk and dieting: how much do they matter for body dissatisfaction and disordered eating behaviors in adolescent girls?.. Journal of Adolescent Health, 47(3), , pp. 270-276..

Nichter, M. \&. V. N., 1994. Fat talk: Body image among adolescent girls., Rutgers university, NJ.: s.n.

O'hara, M. W., 2009. Postpartum depression: what we know.. Journal of clinical psychology.

Park, S., 2005. The influence of presumed media influence on women's desire to be.

Pennington, M. T. a. B., 1990. The development of gender differences in body-size dissatisfaction.. s.1.:s.n.

Pike, K. M. \&. R. J., 1991. Mothers, daughters, and disordered eating. Journal of abnormal psychology, 100(2), 198.. Journal of Abnormal Psychology .

Polivy, J. G. D. M. \&. G. P. E., 1986. In Physical appearance, stigma, and social behavior. In: Causes and consequences of the current preference for thin female physiques.. Erlbaum, Hillsdale, NJ.: s.n., pp. 89-112.

Pomerleu, n.d. 1995: s.n.

RAIL, E. A. H. \&. G., 2012. Contesting "Silhouettes of a Pregnant Belly": Young Pregnant Women's Discursive Constructions of the Body.

Rheanna N. Ata, A. B. L. \&. M. M. L., 2006. The Effects of Gender and Family, Friend, and Media Influences on Eating Behaviors and Body Image During Adolescence. Journal of Youth and Adolescence 36(8):1024-1037 $\cdot$

Rieves, L. \&. C. T. F., 1996. Social developmental factors and women's body-image attitudes. Journal of Social Behavior and Personality, 11(1),

Rodgers, R. F. P. S. J. \&. C. H., 2009. Effects of parental comments on body dissatisfaction and eating disturbance in young adults: A sociocultural model.. s.l.:s.n.

Sorell, G. T. \&. N. C. A., 1981. The role of physical attractiveness as a contributor to individual development. Individuals as producers of their development: A life-span perspective,. s.l.:s.n.

Strang, V. R. \&. S. P. L., 1985. Body Image Attitude During Pregnancy and the Postpartum Period. Journal of Obstetric, Gynecologic, \& Neonatal Nursing.

Swami, V. A. N. C. B. A. P. L. M. \&. T. T., 2009. Are there ethnic differences in positive body image among female British undergraduates?.. In: European Psychologist, 14(4), 288-296.. s.1.:s.n.

Swarr, A. E. \&. R. M. H., 1996. Longitudinal effects of adolescent girls' pubertal development, perceptions of pubertal timing, and parental relations on eating problems. . In: Developmental Psychology, 32(4),. s.1.:s.n.

Tantleff-Dunn, S. \&. G. J. L., 2002. Interpersonal influences on body image development.. Body image: A handbook of theory, research, and clinical practice, 108-116..

Thelen, M. H. \&. C. J. F., 1996. Desire to be thinner and weight control among children and their parents.. Behavior Therapy, 26(1), 85-99.

Thomas F. Cash, T. P., 2002. BODY IMAGE- A Handbook of Theory. s.1.:s.n.

Thompson, J. \&. C. G. E., 2007. The muscular ideal: Psychological, social, and medical perspectives.. s.1., s.n.

Thompson, J. K. H. L. J. A. M. \&. T.-D. S., 1999. Exacting beauty: Theory, assessment, and treatment of body image disturbance. American Psychological Association..

Tiggemann, M. \&. L. J. E., 2001. Body image across the life span in adult women: the role of selfobjectification. Developmental psychology,.

Trampe, D. S. D. A. \&. S. F. W. (., 2007. On models and vases: body dissatisfaction and proneness to social comparison effects.. Journal of personality and social psychology, 92(1), 106. 
Trisolini, E., n.d. History, Herstory, Hairstory:A History and Analysis of Body and Pubic Hair Removal in the United States.

Ussher, J. M. (., 1993. Psychological perspectives on sexual problems: New directions in theory and practice. s.1.:Psychology Press.

Wachs, S. L. D. a. F. L., 2004. "Getting Your Body Back": Postindustrial Fit Motherhood in Shape Fit Pregnancy Magazine. Gender and Society, Vol. 18, pp. 610-624.

Wachs, S. L. D. a. F. L., 2004. "Getting Your Body Back": Postindustrial Fit Motherhood in Shape Fit Pregnancy Magazine.

Wolf, N., 1991. The beauty Myth. s.l.:s.n.

Wolf, N., 1991. The Beauty Myth. s.l.:s.n.

Zimik, C., 2016. Women and Body Image: A Sociological Study of Women in India. International Journal of English language, Literature and Humanities . 